\title{
Association of Polymorphisms of Genes Involved in Lipid Metabolism with Blood Pressure and Lipid Values in Mexican Hypertensive Individuals
}

\author{
Blanca Estela Ríos-González, ${ }^{1,2}$ Bertha Ibarra-Cortés, ${ }^{3}$ Guadalupe Ramírez-López, \\ José Sánchez-Corona, ${ }^{5}$ and María Teresa Magaña-Torres ${ }^{1}$ \\ ${ }^{1}$ División de Genética, Centro de Investigación Biomédica de Occidente, Instituto Mexicano del Seguro Social, \\ Sierra Mojada No. 800, Colonia Independencia, 44340 Guadalajara, JAL, Mexico \\ ${ }^{2}$ Centro Universitario de Ciencias de la Salud, Universidad de Guadalajara, Sierra Mojada No. 950, \\ Colonia Independencia, 44348 Guadalajara, JAL, Mexico \\ ${ }^{3}$ Instituto de Genética Humana "Enrique Corona Rivera", Centro Universitario de Ciencias de la Salud, Universidad de Guadalajara, \\ Sierra Mojada No. 950, Colonia Independencia, 44348 Guadalajara, JAL, Mexico \\ ${ }^{4}$ Unidad de Investigación Epidemiológica y en Servicios de Salud del Adolescente, Instituto Mexicano del Seguro Social, \\ Avenida Tonalá 121, 45400 Tonalá, JAL, Mexico \\ ${ }^{5}$ División de Medicina Molecular, Centro de Investigación Biomédica de Occidente, Instituto Mexicano del Seguro Social, \\ Sierra Mojada No. 800, Colonia Independencia, 44340 Guadalajara, JAL, Mexico \\ Correspondence should be addressed to María Teresa Magaña-Torres; maganamt@yahoo.com.mx
}

Received 18 September 2014; Revised 12 November 2014; Accepted 13 November 2014; Published 21 December 2014

Academic Editor: Claudio Letizia

Copyright (C) 2014 Blanca Estela Ríos-González et al. This is an open access article distributed under the Creative Commons Attribution License, which permits unrestricted use, distribution, and reproduction in any medium, provided the original work is properly cited.

\begin{abstract}
Hypertension and dyslipidemia exhibit an important clinical relationship because an increase in blood lipids yields an increase in blood pressure (BP). We analyzed the associations of seven polymorphisms of genes involved in lipid metabolism (APOA5 rs3135506, APOB rs1042031, FABP2 rs1799883, LDLR rs5925, LIPC rs1800588, LPL rs328, and MTTP rs1800591) with blood pressure and lipid values in Mexican hypertensive (HT) patients. A total of $160 \mathrm{HT}$ patients and 160 normotensive individuals were included. Genotyping was performed through PCR-RFLP, PCR-AIRS, and sequencing. The results showed significant associations in the HT group and HT subgroups classified as normolipemic and hyperlipemic. The alleles FABP2 p.55T, LIPC - 514T, and MTTP -493T were associated with elevated systolic BP. Five alleles were associated with lipids. $L P L$ p.474X and FABP2 p.55T were associated with decreased total cholesterol and LDL-C, respectively; APOA5 p.19W with increased HDL-C; APOA5 p.19W and FABP2 p.55T with increased triglycerides; and $A P O B$ p.4181K and $L D L R$ c.1959T with decreased triglycerides. The $A P O B$ p.E4181K polymorphism increases the risk for HT $(\mathrm{OR}=1.85,95 \% \mathrm{CI}: 1.17-2.93 ; P=0.001)$ under the dominant model. These findings indicate that polymorphisms of lipid metabolism genes modify systolic BP and lipid levels and may be important in the development of essential hypertension and dyslipidemia in Mexican HT patients.
\end{abstract}

\section{Introduction}

Hypertension and dyslipidemia are major global public health problems because they are highly prevalent, contribute to the development of cardiovascular disease, and are components of metabolic syndrome. In Mexico, $26.6 \%$ of the working-age population is hypertensive (HT), $48.7 \%$ exhibits high total cholesterol (TC) levels $(\geq 200 \mathrm{mg} / \mathrm{dL}$ ), and $57.3 \%$ displays high triglyceride (TG) concentrations ( $\geq 150 \mathrm{mg} / \mathrm{dL}$ ) [1]. Essential hypertension (EH) is a multifactorial disease, whose development involves environmental and genetic factors. A significant percentage $(30 \%-60 \%)$ of blood pressure (BP) variation is due to genetic factors, and at least 69 genes have been associated with $\mathrm{EH}[2]$.

A group of genes that have been less well studied but may play an important role in $\mathrm{EH}$ development are those encoding 
proteins involved in lipid metabolism. Hypertension and dyslipidemia show an important clinical relationship because an increase in nonesterified fatty acids (NEFAs) (lipoprotein metabolism products) increases BP, but, in normal conditions, the endocrine, sympathetic, and parasympathetic systems stabilize BP [3]. However, in the presence of chronic hyperlipidemia, such regulatory mechanisms are insufficient, and the sustained increase in NEFAs directly affects cardiac output and peripheral resistance, which are important hemodynamic variables that regulate BP. Cardiac output can be altered by a decrease in the sensitivity of baroreceptors, increased synthesis of catecholamine, and an increased heart rate $[3,4]$, while peripheral resistance may increase by alpha-1 adrenergic receptor hypersensitivity [5], endothelial dysfunction, and atherosclerotic plaque formation [6].

Considering the close relationship between BP and blood lipid levels, we selected seven polymorphisms of seven genes that have mainly been associated with cardiovascular disease, atherosclerosis, dyslipidemias, and obesity. (1) APOA5 p.S19W (rs3135506): the APOAV protein plays an important role in triglyceride (TG) metabolism, and the p.19W allele is negatively correlated with the secretion of this protein [7] and has been associated with increased TG and high-density lipoprotein cholesterol (HDL-C) levels [8, 9]. (2) $A P O B$ p.E4181K (rs1042031): the APOB protein is the main apolipoprotein found in chylomicrons (CM) and low-density cholesterol lipoproteins (LDL-C). The p.4181K allele increases LDL-C catabolism; therefore, it is associated with low TC, LDL-C, and APOB protein levels [10]. (3) FABP2 p.A55T (rs1799883): the FABP2 protein is involved in fatty acid use in the intestine, and the p.55T allele shows a higher affinity for fatty acids; hence, it promotes TG-rich lipoprotein formation [11]. (4) LDLR c.1959C>T (rs5925): the LDL receptor is involved in cholesterol homeostasis through lipoprotein endocytosis, and the c.1959T allele is associated with low TC, LDL-C, HDL-C, and TG in different groups of individuals $[12,13]$. (5) LIPC $-514 \mathrm{C}>\mathrm{T}$ (rs1800588): the LIPC protein functions as both a lipase and ligand, and the $-514 \mathrm{~T}$ allele has been positively correlated with HDL-C levels and negatively correlated with LIPC activity [14]. (6) LPL p.S474X (rs328): the LPL protein hydrolyses TG from very low-density lipoprotein cholesterol (VLDL-C) and CM. The mutated allele increases this enzymatic activity; hence, it improves TG-rich lipoprotein depuration [15]. (7) MTTP $-493 \mathrm{G}>\mathrm{T}$ (rs1800591): MTTP is involved in VLDL-C and $\mathrm{CM}$ assemblage and secretion, and the homozygous genotype -493 TT has been associated with low TC, LDL-C, APOB, and MTTP mRNA levels [16].

The aim of the current study was to analyze the relationship of the $A P O A 5$ p.S19W, $A P O B$ p.E4181K, FABP2 p.A55T, LDLR c.1959C> T, LIPC -514 C> T, LPL p.S474X, and MTTP $-493 \mathrm{G}>\mathrm{T}$ polymorphisms with blood pressure and lipid levels in Mexican HT patients and in subgroups classified according to the presentation of different dyslipidemias.

\section{Materials and Methods}

2.1. Study Population. A total of 716 unrelated individuals aged 19-81 years were recruited from Family Medicine Unit number 93 and the Centro de Investigación Biomédica de Occidente of the Instituto Mexicano del Seguro Social in the metropolitan area of Guadalajara, Jalisco, Mexico. Blood samples were drawn from each of the subjects after at least 12 hours of fasting and without drinking alcohol for the preceding 72 hours. Two tubes of blood were collected; the first was used for biochemical testing, and the second was used to extract DNA. Biochemical measurements were performed using enzymatic methods with commercial kits and a semiautomatic ALS 2000 spectrophotometer. Teco diagnostics (Anaheim, CA), Trinder GOD-POD, Spinreact (Esteve de Bas, Girona, Spain), and AccuBind kits (Lake Forest, CA) were employed to determine the lipid profile (TC, LDL-C (using the Friedewald formula), HDL-C, and TG), glucose, and insulin, respectively. Blood pressure was measured according to the criteria proposed by NOM-030SSA2-2009 [17].

From the total subjects $(n=716)$, we selected 320 individuals, mainly considering their blood pressure values, personal clinical history, age, and sex. The subjects included in the study consisted of $160 \mathrm{HT}$ patients with systolic blood pressure $\geq 140 \mathrm{~mm} \mathrm{Hg}$ and/or diastolic blood pressure $\geq 90 \mathrm{~mm} \mathrm{Hg}$ [17] (121 women and 39 men), with a mean age of $47 \pm 10$ years (range 23-69 years), and 160 normotensive subjects (NT) with systolic blood pressure $<140 \mathrm{~mm} \mathrm{Hg}$ and diastolic blood pressure $<90 \mathrm{~mm} \mathrm{Hg}$ [17] (114 women and 46 men), with a mean age of $47 \pm 11$ years (range $20-81$ years). Both groups were paired by 10 -year age range and by sex, and no significant differences were identified based on the age $(P=0.69)$ or sex $(P=0.69)$ variables. None of the included individuals fulfilled all of the criteria for metabolic syndrome. The protocol was designed based on the guidelines outlined in the Declaration of Helsinki. It was reviewed and approved by the institute's ethics committee. The participants were informed of the study objectives and signed a consent letter.

2.2. Molecular Analysis. DNA was extracted from peripheral blood using standard procedures. Polymorphism genotyping was performed by means of three techniques: (a) polymerase chain reaction-restriction fragment length polymorphism (PCR-RFLP) analysis, to identify the $A P O B$ p.E4181K, FABP2 p.A55T, $L D L R$ c.1959C>T, and LPL p.S474X sites; (b) artificial introduction of restriction sites (PCR-AIRS), to detect APOA5 p.S19W and MTTP -493G>T; and (c) sequencing, for the $L I P C-514 \mathrm{C}>\mathrm{T}$ site. The primers used to identify the MTTP $-493 \mathrm{G}>\mathrm{T}$ polymorphism were designed with Oligo 6.0 software (forward $5^{\prime}$ CTA GTG TGC TAA TGA CAG ACA ATG $C 3^{\prime}$ and reverse $5^{\prime}$ GGA TTT AAA TTT AAA CTG TTA ATT CAT ATC AC $3^{\prime}$ ). A 155 bp fragment was amplified and digested with the Hph I enzyme. The PCR mixture included $13.3 \mathrm{ng} / \mu \mathrm{L}$ genomic DNA, $0.46 \mathrm{pg} / \mu \mathrm{L}$ of each primer, $0.204 \mathrm{mM}$ of each dNTP, $1.5 \mathrm{mM} \mathrm{Mg}_{2} \mathrm{Cl}$, and $0.04 \mathrm{U} / \mu \mathrm{L}$ Taq polymerase. The final reaction volume was $15 \mu \mathrm{L}$. The amplification program was as follows: initial denaturation for $4 \mathrm{~min}$ at $95^{\circ} \mathrm{C}$, followed by 30 cycles of denaturation at $95^{\circ} \mathrm{C}$ for $25 \mathrm{sec}$, annealing at $91^{\circ} \mathrm{C}$ for $40 \mathrm{sec}$, and extension at $72^{\circ} \mathrm{C}$ for $45 \mathrm{sec}$, with a final extension at $72^{\circ} \mathrm{C}$ for $7 \mathrm{~min}$.

The PCR and amplification conditions for the remaining six sites were described elsewhere [18]. FABP2 p.A55T, 
which was previously identified through sequencing [18], was genotyped in this work using the restriction enzyme Hha I. The samples employed as digestion controls were confirmed through sequencing in an ABI PRISM 310 sequencer using the BigDye Terminator v3.1 kit and following the manufacturer's instructions.

2.3. Statistical Analyses. The genotypic and allelic frequencies for the seven polymorphisms were established through direct counting. The frequencies were compared among the groups (NT and HT) using the chi-square or Fisher exact test. The effect of the polymorphisms on BP and lipid profile levels was determined in the group of HT patients and the HT subgroups classified as normolipemic and hyperlipemic according to lipid profile. An analysis of variance (ANOVA) was performed for the genotypes, followed by post hoc tests (Bonferroni or Dunnett, in relation to variance homogeneity). The mean differences between alleles were calculated with Student's $t$-test. The Pearson (for quantitative and normally distributed variables) and Spearman (for qualitative variables) correlations and multiple linear regression models with genotypes and alleles were employed. Furthermore, to estimate the relationships of the seven polymorphisms with hypertension and different dyslipidemias, multiple logistic regression models were generated. A value of $P<0.05$ was considered statistically significant. The analyses were performed using SPSS v.18.0.

\section{Results}

3.1. Genotypic and Allelic Frequencies. The genotype and allele frequency distributions of the seven polymorphisms are shown in Table 1. All three possible genotypes were observed at each site and exhibited heterogeneous frequencies. The frequencies of the mutant genotypes of FABP2 p.A55T, $L D L R$ c. $1959 \mathrm{C}>\mathrm{T}$, and $L I P C-514 \mathrm{C}>\mathrm{T}$ were approximately $30 \%$ in the HT and NT groups. The four remaining polymorphisms displayed frequencies lower than $5 \%$. In both of the studied groups, the mutant alleles of LDLR c.1959T and LIPC -514T were more frequent than the wild-type alleles; in contrast $L P L$ p.474X mutant allele did not exceed a 10\% frequency.

Comparison of the genotypic and allelic frequencies between the groups revealed statistically significant differences only for the $A P O B$ p.E4181K polymorphism $(P=$ 0.01 and $P=0.04$, resp.) due to a higher percentage of heterozygotes in the HT group (Table 1).

3.2. Effect of the Polymorphisms on the Lipid Profile and Blood Pressure of HT Patients. In the association analysis between the polymorphisms versus the variables, 70 patients from the total HT group receiving antihypertensive and/or hypolipidemic treatments were excluded.

Considering all of the HT patients not receiving drug treatment ( $n=90)$, the following significant associations with TC and TG were observed. Subjects with the LPL p.474X allele showed lower TC concentrations $(185.0 \mathrm{mg} / \mathrm{dL})$ than the patients with the p.474S allele $(203.0 \mathrm{mg} / \mathrm{dL}, P=0.006)$, while individuals with the FABP2 p.55T allele exhibited higher TG concentrations $(161.2 \mathrm{mg} / \mathrm{dL})$ than the patients with the p.55A allele $(131.2 \mathrm{mg} / \mathrm{dL} ; P=0.006)$. A significant multiple linear regression model with the FABP2 p.55T and LDLR c.1959T alleles to explain TG levels was established (Table 4).

In regard to blood pressure, subjects carrying the LIPC $-514 \mathrm{~T}$ allele showed higher SBP values $(151.2 \mathrm{~mm} \mathrm{Hg})$ than patients with the $-514 \mathrm{C}$ allele $(146.2 \mathrm{~mm} \mathrm{Hg}), P=0.039$.

3.3. Hypertensive Patients Classified as Normolipemic and Hyperlipemic: Effect of the Polymorphisms on the Lipid Profile and Blood Pressure. All of the studied polymorphisms are located in genes that modulate lipid metabolism and have been associated mainly with dyslipidemia and/or cardiovascular disease. Dyslipidemias distribution in HT patients is shown in Table 2 and the classification was done according the NOM-037-SSA2-2002 [19].

Association analysis was performed in two subgroups of hypertensive patients, normolipemic (without dyslipidemia) and hyperlipemic (with dyslipidemia) (Table 2). Normolipemic subgroup was integrated by 50 patients, with a mean age of $50 \pm 11$ years (range 30-69 years). Hyperlipemic subgroup was formed by 40 patients, with a mean age of $45 \pm 11$ years (23-69 years). Age variable was not significantly different between subgroups $(P=0.60)$.

Analysis of the two subgroups showed many significant results. However, we present only those for which a multiple linear regression model was generated and showed significant values for ANOVA, Student's $t$-test, or Spearman correlation. Table 3 provides the ANOVA, Student's $t$-test, and Spearman correlation results; two ANOVA nonsignificant $P$ values were included to indicate trends (FABP2 p.55T allele for TG and SBP with $P=0.09$ and $P=0.08$, resp.). The multiple linear regression models for each polymorphism and quantitative variable are presented in Table 4 . To avoid duplicate data, the values of the association tests are not provided in the text and are only shown in tables.

APOA5 p.S19W. The p.19W allele was associated with significant increases in HDL-C and TG in normolipemic and hyperlipemic HT patients, respectively.

$A P O B$ p.E4181K. We detected one significant association in the normolipemic subgroup; the HT patients carrying the p. $4181 \mathrm{~K}$ allele showed a lower TG concentration than those bearing the p.4181E allele.

FABP2 p.A55T. This polymorphism exhibited an association with three variables. TG and SBP levels were increased significantly in presence of the mutated allele p.55T in hyperlipemic and normolipemic subgroups, respectively; while LDL-C values were lower in hyperlipemic patients with $\mathrm{p} .55 \mathrm{~T}$ versus p.55A.

LPL p.S474X C>G. The p.474X allele was associated with lower TC levels in HT hyperlipemic patients.

MTTP-493 G>T. Hyperlipemic patients carrying the $-493 \mathrm{~T}$ allele showed statistically higher SBP levels than those with the $-493 \mathrm{G}$ allele (Tables 3 and 4). 


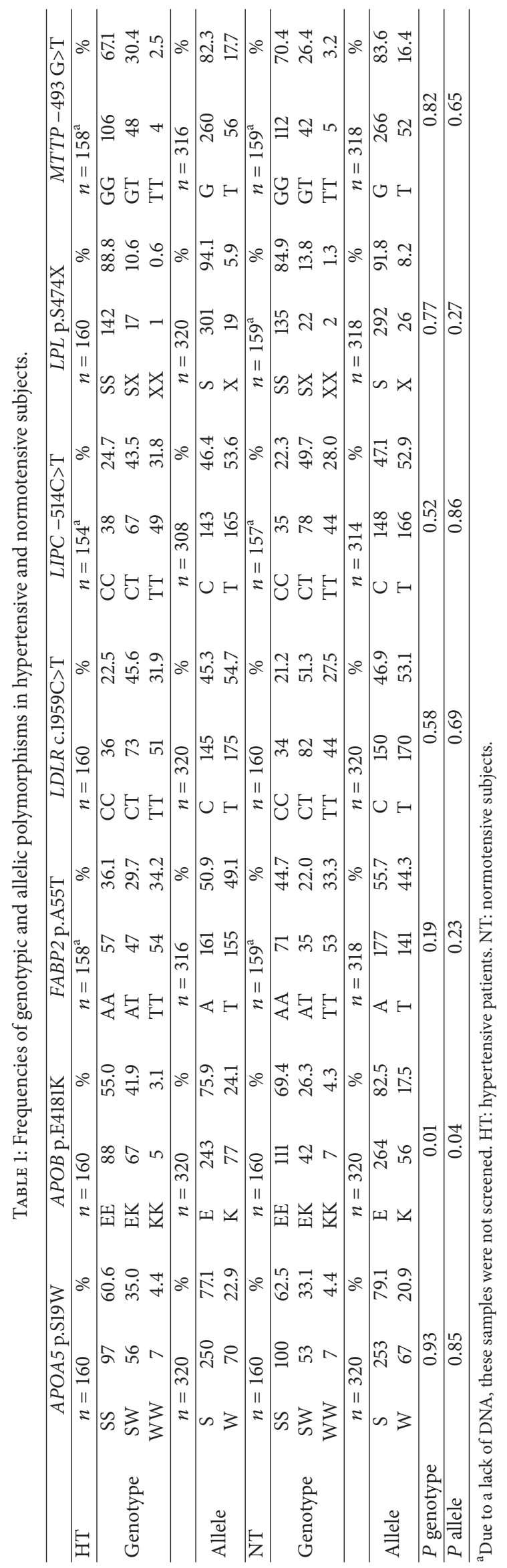


TABLE 2: Distribution of dyslipidemias in hypertensive patients.

\begin{tabular}{lcccc}
\hline & Without & $\%$ & With & $\%$ \\
\hline${ }^{\mathrm{a}}$ Hypercholesterolemia & 66 & 73.3 & 24 & 26.7 \\
b LDL-C $\geq 130 \mathrm{mg} / \mathrm{dL}$ & 51 & 56.7 & 39 & 43.3 \\
${ }^{\mathrm{c}}$ Hypertriglyceridemia & 81 & 90.0 & 9 & 10.0 \\
${ }^{\mathrm{d}}$ Mixed hyperlipidemia & 83 & 92.2 & 7 & 7.8 \\
${ }^{\mathrm{e}}$ Hypoalphalipoproteinemia & 63 & 70.0 & 27 & 30.0 \\
${ }^{\mathrm{f}}$ Dyslipidemia & 50 & 55.6 & 40 & 44.4 \\
\hline
\end{tabular}

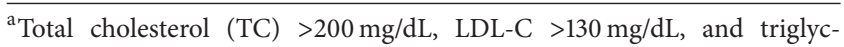
erides (TG) $<200 \mathrm{mg} / \mathrm{dL} ;{ }^{\mathrm{b}} \mathrm{LDL}-\mathrm{C} \geq 130 \mathrm{mg} / \mathrm{dL}$; ${ }^{\mathrm{c}} \mathrm{TC}<200 \mathrm{mg} / \mathrm{dL}$, LDL-C $<130 \mathrm{mg} / \mathrm{dL}$, and $\mathrm{TG}>200 \mathrm{mg} / \mathrm{dL} ;{ }^{\mathrm{d}} \mathrm{TC} \geq 200 \mathrm{mg} / \mathrm{dL}$, LDL-C $\geq 130 \mathrm{mg} / \mathrm{dL}$, and TG $\geq 200 \mathrm{mg} / \mathrm{dL} ;{ }^{\mathrm{e}} \mathrm{HDL}-\mathrm{C}<35 \mathrm{mg} / \mathrm{dL}$; and ${ }^{\mathrm{f}}$ presence of any dyslipidemia (hypercholesterolemia or hypertriglyceridemia or mixed hyperlipidemia).

3.4. Multiple Linear Regression Models. In total, nine distinct multiple linear regression models with statistically significant differences were generated with genotypes and alleles data; in Table 4, the results obtained with the alleles are shown. An integrated model with two polymorphisms FABP2 p.A55T and $L D L R$ c.1959C> T, explaining TG levels, was observed in the total group of hypertensive $(n=90)$ and hyperlipemic subgroup. The eight remaining models included only one polymorphism. The TG variable presented a greater number of significant models (Table 4).

3.5. Logistic Regression Models. In this analysis, we observed two models. The first was observed for $A P O B$ p.E4181K, which increased the risk of developing essential hypertension under the dominant model (EK+KK v. EE) $(\mathrm{OR}=1.85$; 95\% CI: $1.2-$ 2.9 and $P=0.01)$. The second model showed that HT patients carrying the APOA5 p.19W allele exhibit an increased risk of presenting mixed hyperlipidemia $(\mathrm{OR}=8.8$; 95\% CI: $1.76-$ $44.0 ; P=0.02)$ under the recessive model (WW v. SW+SS).

\section{Discussion}

Essential hypertension is a multifactorial disease with a strong genetic component; therefore, genes corresponding to different metabolic routes have been explored. Because increased blood lipid levels are known to result in an increase in blood pressure, in the present work, we analyzed seven polymorphisms of genes involved in lipid metabolism in HT patients to examine their role in the development of essential hypertension and dyslipidemias.

It is important to note that when the association analysis was performed in the entire HT group, only a few significant results were observed. However, the analysis of the HT patients classified according to lipid levels revealed more significant associations between the polymorphisms and diverse variables. Studies examining these polymorphisms in different populations have revealed heterogeneous results. A possible explanation is that these differences occur because most of the authors did not analyze the data by separating individuals according to dyslipidemia.

4.1. Genotypic and Allelic Frequencies. In the genotypic and allelic frequency distribution analyses for the HT and NT groups, we observed significant differences for the polymorphism $A P O B$ p.E4181K, based on the presence of more heterozygotes in the HT group (41.9\% versus $23.3 \%$ ). Other studies have analyzed this polymorphism in relation to cardiovascular disease, but the authors did not observe significant differences in the genotype and/or allele distribution $[20,21]$

We compared the results for the seven polymorphisms with the reported frequencies in the general Mexican population $[18,22]$. The analysis showed similar genotypic frequency distributions for the general population and NT individuals $(P>0.05)$. However, we observed significant differences between the general population and the HT group for three polymorphisms: the $A P O B$ p.E4181K site $(P=0.008), F A B P 2$ p.A55T $(P=0.002)$, and the $L I P C-514 \mathrm{C}>\mathrm{T}$ polymorphism $(P=0.04)$. Only two of the seven analyzed polymorphisms have been studied in HT patients: LDLR c.1959C > T (Chinese population) [12] and LPL p.S474X (Chinese and Caucasian populations) [23]; similar to the results of the present study, the authors did not report significant differences in the genotypic and allelic frequency distribution.

4.2. Distribution of Dyslipidemias in HT Patients. In Mexico, dyslipidemias and essential hypertension are the most common risk factors for the development of cardiovascular disease in the general adult population [24, 25]. In this study more than $40 \%$ of the subjects exhibited some form of dyslipidemia (hypercholesterolemia or hypertriglyceridemia or mixed hyperlipidemia), which indicates a significant health problem. Various reports have revealed that one of the most frequent dyslipidemias in the Mexican population is hypoalphalipoproteinemia, and similar results were obtained in this study. Low HDL-C levels in HT patients have also been observed in other populations at frequencies higher than $30 \%$ [26]. Although a clinical finding of hypoalphalipoproteinemia does not represent a health problem by itself, it is an important risk factor for developing cardiovascular disease and metabolic syndrome when associated with high TG and TC levels. Furthermore, in patients with hypertension, several coincident biochemical alterations can increase cardiovascular risk and complicate hypertension management.

\subsection{Effect of the Polymorphisms on the Lipid Profile and Blood Pressure of Hypertensive Patients and Normolipemic and Hyperlipemic Subgroups}

APOA5 p.S19W. The APOAV protein is a component of the lipoproteins HDL-C, VLDL-C, and CM; it activates LPL for efficient TG lipolysis. In this study, the p.19W allele was associated with increased HDL-C in normolipemic HT patients (Tables 3 and 4). This association between high HDL$\mathrm{C}$ levels and the p.19W allele has been reported in healthy subjects from Puerto Rico [8].

High TG levels associated with the p.19W allele were observed in hyperlipemic HT patients. Similar results have been detected in young, healthy Caucasian males [9], Spanish subjects in the ICARIA project [27], and Caucasian children 6-8 years old [28]. This association was also reflected in 
TABLE 3: Results of ANOVA, Student's $t$, and correlation tests between polymorphisms and quantitative variables in normolipemic and hyperlipemic hypertensive patients.

\begin{tabular}{|c|c|c|c|c|c|c|}
\hline \multicolumn{7}{|c|}{ APOA5 p.S19W } \\
\hline & Variable & Genotype SS & Genotype WW & $P$ & \multicolumn{2}{|c|}{ Subgroup } \\
\hline \multirow{4}{*}{ ANOVA } & HDL-C (mg/dL) & 41.6 & 67.0 & 0.000 & \multicolumn{2}{|c|}{ Normolipemic } \\
\hline & HDL-C (mg/dL) & $49.5^{\mathrm{SW}}$ & 67.0 & 0.001 & \multicolumn{2}{|c|}{ Normolipemic } \\
\hline & TG (mg/dL) & 172.0 & 221.0 & 0.032 & \multicolumn{2}{|c|}{ Hyperlipemic } \\
\hline & & Allele S & Allele W & $P$ & Correlation & $P$ \\
\hline \multirow{2}{*}{ Student's $t$-test } & HDL-C (mg/dL) & 43.6 & 52.3 & 0.007 & 0.361 & 0.010 \\
\hline & TG (mg/dL) & 183.5 & 235.5 & 0.023 & 0.315 & 0.048 \\
\hline \multicolumn{7}{|c|}{$A P O B$ p.E4181K } \\
\hline & Variable & Genotype EE & Genotype EK & $P$ & \multicolumn{2}{|c|}{ Subgroups } \\
\hline \multirow[t]{2}{*}{ ANOVA } & TG (mg/dL) & 121.6 & 88.3 & 0.006 & \multicolumn{2}{|c|}{ Normolipemic } \\
\hline & & Allele E & Allele K & & Correlation & $\boldsymbol{P}$ \\
\hline Student's $t$-test & TG (mg/dL) & 114.6 & 83.2 & 0.003 & -0.435 & 0.002 \\
\hline \multicolumn{7}{|c|}{$F A B P 2$ p.A55T } \\
\hline & Variable & Genotype AA & Genotype TT & $P$ & \multicolumn{2}{|c|}{ Subgroups } \\
\hline \multirow{4}{*}{ ANOVA } & LDL-C & 170.2 & 135.9 & 0.018 & \multicolumn{2}{|c|}{ Hyperlipemic } \\
\hline & TG (mg/dL) & 164.3 & 237.2 & $0.090^{\mathrm{a}}$ & \multicolumn{2}{|c|}{ Hyperlipemic } \\
\hline & $\mathrm{SBP}(\mathrm{mm} \mathrm{Hg})$ & 145.0 & 156.0 & $0.082^{\mathrm{a}}$ & \multicolumn{2}{|c|}{ Normolipemic } \\
\hline & & Allele A & Allele T & $P$ & Correlation & $P$ \\
\hline \multirow{3}{*}{ Student's $t$-test } & LDL-C & 162.2 & 141.5 & 0.018 & -0.328 & 0.042 \\
\hline & TG (mg/dL) & 169.2 & 216.5 & 0.006 & 0.382 & 0.017 \\
\hline & $\mathrm{SBP}(\mathrm{mm} \mathrm{Hg})$ & 147.1 & 155.0 & 0.007 & 0.346 & 0.026 \\
\hline \multicolumn{7}{|c|}{ LPL p.S474X } \\
\hline & Variable & Genotype SS & Genotype SX & $P$ & \multicolumn{2}{|c|}{ Subgroups } \\
\hline \multirow[t]{2}{*}{ ANOVA } & $\mathrm{TC}(\mathrm{mg} / \mathrm{dL})$ & 236.0 & 189.4 & 0.035 & \multicolumn{2}{|c|}{ Hyperlipemic } \\
\hline & & Allele S & Allele X & $P$ & Correlation & $P$ \\
\hline Student's $t$-test & $\mathrm{TC}(\mathrm{mg} / \mathrm{dL})$ & 234.1 & 189.6 & 0.039 & -0.335 & 0.035 \\
\hline \multicolumn{7}{|c|}{ MTTP -493 G> T } \\
\hline & Variable & Genotype GG & Genotype GT & $P$ & \multicolumn{2}{|c|}{ Subgroups } \\
\hline \multirow[t]{2}{*}{ ANOVA } & $\mathrm{SBP}(\mathrm{mm} \mathrm{Hg})$ & 141.5 & 154.5 & 0.023 & \multicolumn{2}{|c|}{ Hyperlipemic } \\
\hline & & Allele G & Allele T & $P$ & Correlation & $P$ \\
\hline Student's $t$-test & $\mathrm{SBP}(\mathrm{mm} \mathrm{Hg})$ & 144.2 & 154.5 & 0.003 & 0.364 & 0.023 \\
\hline
\end{tabular}

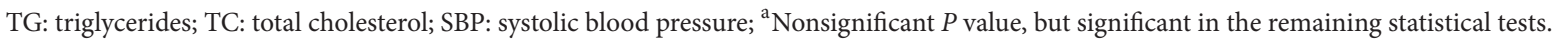

TABLE 4: Multiple linear regression models observed in normolipemic and hyperlipemic hypertensive patients.

\begin{tabular}{|c|c|c|c|c|c|}
\hline Variable & Allele & Constant & $B$ & $P$ & Subgroups \\
\hline $\mathrm{TC}(\mathrm{mg} / \mathrm{dL})$ & LPL p.474X & 234.1 & -44.5 & 0.039 & Hyperlipemic \\
\hline LDL-C (mg/dL) & $F A B P 2$ p.55T & 162.2 & -20.7 & 0.024 & Hyperlipemic \\
\hline HDL-C (mg/dL) & APOA5 p.19W & 43.6 & 8.7 & 0.007 & Normolipemic \\
\hline \multirow{7}{*}{ TG (mg/dL) } & APOA5 p.19W & 183.5 & 52.0 & 0.023 & Hyperlipemic \\
\hline & $A P O B$ p. $4181 \mathrm{~K}$ & 114.6 & -31.4 & 0.003 & Normolipemic \\
\hline & $F A B P 2$ p.55T & 169.2 & 47.3 & 0.010 & Hyperlipemic \\
\hline & $F A B P 2$ p.55T & \multirow{2}{*}{142.4} & 36.5 & 0.002 & \multirow{2}{*}{ Hypertensive } \\
\hline & LDLR c.1959T & & -26.3 & 0.024 & \\
\hline & $F A B P 2$ p.55T & \multirow{2}{*}{190.0} & 53.2 & 0.003 & \multirow{2}{*}{ Hyperlipemic } \\
\hline & LDLR c.1959T & & -43.0 & 0.017 & \\
\hline \multirow{2}{*}{ SBP (mmHg) } & $F A B P 2$ p.55T & 147.1 & 7.9 & 0.007 & Normolipemic \\
\hline & $M T T P-493 \mathrm{~T}$ & 144.2 & 10.3 & 0.042 & Hyperlipemic \\
\hline
\end{tabular}

TC: total cholesterol; TG: triglycerides; SBP: systolic blood pressure. 
the logistic regression analysis because, in the HT group, the p.19W allele (under recessive model) increased the risk of presenting mixed hyperlipidemia by more than 8 -fold.

In general, the findings of increased HDL-C and TG are consistent with the function of the APOA5 p.19W variant because reduced secretion of the protein decreases LPL activity [7], increasing TG levels in plasma. Moreover, APOAV is a component of HDL-C, and APOAV deficiency may reduce the metabolism of these particles, which increases their blood concentration.

$A P O B$ p.E4181K. APOB is the primary protein found in VLDL-C, IDL-C, and LDL-C; thus, it is important for cholesterol homeostasis. The $A P O B$ p.E4181K polymorphism is located in the proximal portion of the terminal carboxyl and increases LDL-C catabolism, with a consequent decrease of LDL-C and APOB levels [10]. In the present study, the p.4181K allele was associated with lower TG levels in normolipemic HT patients; this association has not been observed in other populations. The association between low TC and LDL$\mathrm{C}$ levels and the p.4181K allele has been demonstrated in most populations studied to date. However, the literature is inconsistent regarding whether the $\mathrm{p} .4181 \mathrm{~K}$ allele is protective or is a risk factor for cardiovascular diseases, as both models have been observed [10]. In this work we found a risk of developing hypertension conferred by $\mathrm{p} .4181 \mathrm{~K}$ allele, with an OR $=1.85 ; 95 \% \mathrm{CI}: 1.2-2.9 ; P=0.01$, which is similar to the findings of a meta-analysis of 30 case-control reports where the risk of developing cardiovascular disease and myocardial infarction was evaluated, and the authors obtained an OR of 1.73 (95\% CI: 1.19-2.50) [29]. Thus, we suggest that the p. $4181 \mathrm{~K}$ allele increases the risk of developing cardiovascular disease, myocardial infarction, and hypertension; however, its pathway of action must be different from that related to lipids because this allele is associated with lower TG, TC, and LDLC concentrations.

FABP2 p.A55T. The FABP2 p.A55T polymorphism has been extensively studied and referenced in the literature. The p.55T allele results in a higher affinity of this protein for longchain fatty acids and, hence, greater absorption of such fatty acids [11]. In this study, the p.55T allele was associated with increased SBP in normolipemic HT patients, which differs from the findings of de Luis, who observed a decrease in SBP in nondiabetic obese subjects [30]. However, an association between the mutated allele and an increase in TG was observed in the HT patients and hyperlipemic subgroup and has been reported previously [31, 32]. This association is consistent with the increase in fatty acid absorption found in individuals with the p.55T allele and therefore enhances TGrich lipoprotein formation [11]. Moreover, the studied groups in which this association had been observed also exhibit low $\mathrm{HDL}-\mathrm{C}$, as found in our patients with hypoalphalipoproteinemia.

LDLR c.1959C>T. The c.1959T allele was associated with low TG levels in HT patients and hyperlipemic subgroup, in a multiple linear regression model (Table 4). In general population of China, this allele has been associated with lower TC, LDL-C, and TG concentrations $[12,13]$.

LIPC $-514 C>T$. One of the primary functions of hepatic lipase is to hydrolyze TG and phospholipids, and hepatic lipase is an important enzyme in HDL-C metabolism [14]. HT patients group with the $-514 \mathrm{~T}$ allele exhibited higher SBP values compared with those carrying the $-514 \mathrm{C}$ allele. To our knowledge, this is the first study to analyze this polymorphism in HT patients and to assess its association with blood pressure.

LPL p.S474X. The primary function of LPL is to hydrolyze TG from CM and VLDL-C. It has been shown that the p.474X allele increases LPL enzymatic activity; hence, it has been associated with decreased plasma TG and increased HDL$\mathrm{C}$ [15]. In the present work, in the HT and HT hyperlipemic groups, the p.474X allele was associated with lower TC levels, as previously observed in a meta-analysis. The mutated allele has also been associated with lower TG and SBP levels as well as higher HDL-C levels [33]. Consistent with such results, the presence of the p.474X allele has shown in previous reports a lower risk for developing coronary heart disease [33] and hypertension (OR 0.78; 95\% CI: 0.62-0.98, $P=0.03$ ) [34].

MTTP $-493 G>T$. The MTTP enzyme is involved in the assembly and secretion of VLDL-C, which transports TG, cholesterol, and phospholipids [16]. In this study, the $-493 \mathrm{~T}$ allele was associated with increased SBP in HT hyperlipemic subgroup, which has not been previously reported in the literature. However, in healthy males and hypercholesterolemia patients, the mutated allele has been associated with low TC [16] and TG levels [35], respectively.

\section{Conclusions}

We highlight three important conclusions. (i) The $A P O B$ p.E4181K polymorphism (under the dominant model) is associated with an increased risk for hypertension. (ii) Three polymorphisms were found to be associated with systolic blood pressure levels in HT patients. Increased SBP was associated with the FABP2 p.55T, LIPC -514T, and MTTP $-493 \mathrm{~T}$ alleles. (iii) Modifications of the four different lipids studied herein were observed to be correlated with certain polymorphisms. Total cholesterol is decreased in subjects with the LPL p.474X allele; LDL-C is decreased with FABP2 p.55T; HDL-C is increased with APOA5 p.19W; and triglycerides are increased with $A P O A 5$ p.19W and FABP2 p.55T but decreased with $A P O B$ p.4181K and LDLR c.1959T. These findings indicate that polymorphisms of lipid metabolism genes modify systolic blood pressure and lipid levels and may be important for the development of essential hypertension and dyslipidemia in Mexican HT patients.

\section{Conflict of Interests}

The authors declare that there is no conflict of interests regarding the publication of this paper. 


\section{Acknowledgments}

This study was supported by the Consejo Nacional de Ciencia y Tecnología (CONACYT)/Fondos Sectoriales Sector Salud 2005-C02-14410. The authors gratefully acknowledge the investigators of the GEMHAS group: María Cristina Morán Moguel, Javier Perea Díaz, Silvia Esperanza Flores Martínez, Alejandra Guadalupe García Zapién, Caridad Aurea Leal Cortés, José Z. Parra Carrillo, Salvador Fonseca Reyes, José Amado Espinosa Lobato, Alejandro Bravo Cuellar, María Elena Aguilar Aldrete, and Ernesto Sahagún Flores. They would also like to thank Rogelio Troyo Sanroman for assistance with the statistical analysis.

\section{References}

[1] C. Munguía-Miranda, R. G. Sánchez-Barrera, D. HernándezSaavedra, and M. C. Cruz-López, "Prevalencia de dislipidemias en una población de sujetos en apariencia sanos y su relación con la resistencia a la insulina," Salud Pública de México, vol. 50, no. 5, pp. 375-382, 2008.

[2] J. Ran, H. Li, J. Fu et al., "Construction and analysis of the protein-protein interaction network related to essential hypertension," BMC Systems Biology, vol. 7, article 32, 2013.

[3] I. Skrapari, N. Tentolouris, and N. Katsilambros, "Baroreflex function: determinants in healthy subjects and disturbances in diabetes, obesity and metabolic syndrome," Current Diabetes Reviews, vol. 2, no. 3, pp. 329-338, 2006.

[4] C. A. Gadegbeku, A. Dhandayuthapani, Z. E. Sadler, and B. M. Egan, "Raising lipids acutely reduces baroreflex sensitivity," American Journal of Hypertension, vol. 15, no. 6, pp. 479-485, 2002.

[5] K. T. Stepniakowski, T. L. Goodfriend, and B. M. Egan, "Fatty acids enhance vascular $\alpha$-adrenergic sensitivity," Hypertension, vol. 25, no. 4, pp. 774-778, 1995.

[6] N. R. Villela, L. G. Kramer-Aguiar, D. A. Bottino, N. Wiernsperger, and E. Bouskela, "Metabolic disturbances linked to obesity: the role of impaired tissue perfusion," Arquivos Brasileiros de Endocrinologia e Metabologia, vol. 53, no. 2, pp. 238-245, 2009.

[7] P. J. Talmud, J. Palmen, W. Putt, L. Lins, and S. E. Humphries, "Determination of the functionality of common APOA5 polymorphisms," Journal of Biological Chemistry, vol. 280, no. 31, pp. 28215-28220, 2005.

[8] J. Mattei, S. Demissie, K. L. Tucker, and J. M. Ordovas, "Apolipoprotein A5 polymorphisms interact with total dietary fat intake in association with markers of metabolic syndrome in Puerto Rican older adults," Journal of Nutrition, vol. 139, no. 12, pp. 2301-2308, 2009.

[9] S. Martin, V. Nicaud, S. E. Humphries, and P. J. Talmud, "Contribution of APOA5 gene variants to plasma triglyceride determination and to the response to both fat and glucose tolerance challenges," Biochimica et Biophysica Acta (BBA)Molecular Basis of Disease, vol. 1637, no. 3, pp. 217-225, 2003.

[10] M. Benn, B. G. Nordestgaard, J. S. Jensen, and A. TybjærgHansen, "Polymorphisms in apolipoprotein B and Risk of ischemic stroke," Journal of Clinical Endocrinology and Metabolism, vol. 92, no. 9, pp. 3611-3617, 2007.

[11] A. Auinger, U. Helwig, D. Rubin et al., "Human intestinal fatty acid binding protein 2 expression is associated with fat intake and polymorphisms," The Journal of Nutrition, vol. 140, no. 8, pp. 1411-1417, 2010.

[12] A.-P. Liu, S.-Y. Zhan, L.-M. Li et al., "Association between AvaII exon 13 polymorphism at the LDL receptor gene different and serum lipid levels in normotensives and essential hypertensives in Shanghai," Zhonghua Liu Xing Bing Xue Za Zhi, vol. 24, no. 7, pp. 542-546, 2003.

[13] X. J. Long, R. X. Yin, K. L. Li et al., "Low density lipoprotein receptor gene Ava II polymorphism and serum lipid levels in the Guangxi Bai Ku Yao and Han populations," Lipids in Health and Disease, vol. 10, article 34, 2011.

[14] A. Isaacs, F. A. Sayed-Tabatabaei, O. T. Njajou, J. C. Witteman, and C. M. Van Duijn, "The $-514 \mathrm{C} \rightarrow \mathrm{T}$ hepatic lipase promoter region polymorphism and plasma lipids: a meta-analysis," The Journal of Clinical Endocrinology and Metabolism, vol. 89, no. 8, pp. 3858-3863, 2004.

[15] J. Rip, M. C. Nierman, C. J. Ross et al., "Lipoprotein lipase S447X: a naturally occurring gain-of-function mutation," Arteriosclerosis, Thrombosis, and Vascular Biology, vol. 26, no. 6, pp. 1236-1245, 2006.

[16] H. Ledmyr, A. D. McMahon, E. Ehrenborg et al., "The microsomal triglyceride transfer protein gene-493T variant lowers cholesterol but increases the risk of coronary heart disease," Circulation, vol. 109, no. 19, pp. 2279-2284, 2004.

[17] Norma Oficial Mexicana, "Para la prevención, detección, diagnóstico, tratamiento y control de la hipertensión arterial sistémica," NOM-030-SSA2-2009, 2009.

[18] B. E. Ríos-González, K. E. Luévano-Ortega, A. M. SaldañaCruz, J. R. González-García, and M. T. Magaña-Torres, "Polymorphisms of seven genes involved in lipid metabolism in an unselected Mexican population," Journal of Genetics, vol. 90, pp. $1-6,2011$.

[19] Norma Oficial Mexicana, "Para la prevención, tratamiento y control de las dislipidemias," NOM-037-SSA2-2002, 2002.

[20] K. E. Luévano, J. R. González, F. J. Perea, and M. T. Magaña, "Linkage disequilibrium between four MTTP gene polymorphisms in a Mexican population," Annals of Human Biology, vol. 36, no. 2, pp. 211-219, 2009.

[21] S. H. Hong, J. Song, and J. Q. Kim, “The haplotype analyses using multiple markers of the apolipoprotein B gene in patients with coronary artery disease," Journal of Korean Medical Science, vol. 16, no. 6, pp. 719-724, 2001.

[22] M. Scartezini, M. A. Zago, E. A. Chautard-Freire-Maia et al., "The X-X-/E+E+ genotype of the XbaI/EcoRI polymorphisms of the apolipoprotein $\mathrm{B}$ gene as a marker of coronary artery disease in a Brazilian sample," Brazilian Journal of Medical and Biological Research, vol. 36, no. 3, pp. 369-375, 2003.

[23] B. Li, D. Ge, Y. Wang et al., "Lipoprotein lipase gene polymorphisms and blood pressure levels in the Northern Chinese Han population," Hypertension Research, vol. 27, no. 6, pp. 373-378, 2004.

[24] C. A. Aguilar-Salinas, F. J. Gómez-Pérez, J. Rull, S. Villalpando, S. Barquera, and R. Rojas, "Prevalence of dyslipidemias in the Mexican National Health and Nutrition Survey 2006," Salud Publica de Mexico, vol. 52, no. 1, pp. S44-S53, 2010.

[25] S. Barquera, I. Campos-Nonato, L. Hernández-Barrera et al., "Hypertension in mexican adults: results from the National Health and Nutrition Survey 2006," Salud Pública de México, vol. 52, supplement 1, pp. S63-S71, 2010. 
[26] P. Conthe, P. Gómez-Fernández, F. de Álvaro, C. FernándezPérez, J. González-Esteban, and L. Cea-Calvo, "HDL cholesterol and cardiovascular disease in a population with hypertension and type 2 diabetes mellitus. RICARHD study," Revista Clinica Española, vol. 209, no. 5, pp. 227-233, 2009.

[27] M.-J. Ariza, M.-Á. Sánchez-Chaparro, F.-J. Barón et al., "Additive effects of LPL, APOA5 and APOE variant combinations on triglyceride levels and hypertriglyceridemia: Results of the ICARIA genetic sub-study," BMC Medical Genetics, vol. 11, article 66, 2010.

[28] M. Guardiola, J. Ribalta, D. Gómez-Coronado, M. A. Lasunción, M. de Oya, and C. Garcés, "The apolipoprotein A5 (APOA5) gene predisposes Caucasian children to elevated triglycerides and vitamin E (four provinces study)," Atherosclerosis, vol. 212, no. 2, pp. 543-547, 2010.

[29] B. D. Chiodini, S. Barlera, M. G. Franzosi, V. L. Beceiro, M. Introna, and G. Tognoni, "APO B gene polymorphisms and coronary artery disease: a meta-analysis," Atherosclerosis, vol. 167, no. 2, pp. 355-366, 2003.

[30] D. A. de Luis, R. Aller, O. Izaola, M. Gonzalez Sagrado, and R. Conde, "Influence of ALA54THR polymorphism of fatty acid binding protein 2 on lifestyle modification response in obese subjects," Annals of Nutrition and Metabolism, vol. 50, no. 4, pp. 354-360, 2006.

[31] Y. Liu, C. Zhai, P. Cao, Y. Zheng, S. Zhou, and J. Chi, "FABP2 gene polymorphism and metabolic disorder of lipids in the middle aged and aged population," Wei Sheng Yan Jiu, vol. 40, no. 4, pp. 461-464, 2011.

[32] X. S. Wang, H. Bai, P. Fan, R. Liu, Y. Liu, and B. W. Liu, "Analysis of the FABP2 gene Ala54Thr polymorphism in non-obese and obese Chinese," Sichuan Da Xue Xue Bao. Yi Xue Ban, vol. 42, no. 1, pp. 19-23, 2011.

[33] C. Wang, T. Sun, H. Li, J. Bai, and Y. Li, "Lipoprotein lipase Ser447Ter polymorphism associated with the risk of ischemic stroke: a meta-analysis," Thrombosis Research, vol. 128, no. 5, pp. e107-e112, 2011.

[34] W. Q. Niu and Y. Qi, "Meta-based association of the lipoprotein lipase gene S447X variant with hypertension and blood pressure variation," Journal of Human Hypertension, vol. 25, no. 6, pp. 383-390, 2011.

[35] B. Lundahl, T. P. Leren, L. Ose, A. Hamsten, and F. Karpe, "A functional polymorphism in the promoter region of the microsomal triglyceride transfer protein (MTP -493G/T) influences lipoprotein phenotype in familial hypercholesterolemia," Arteriosclerosis, Thrombosis, and Vascular Biology, vol. 20, no. 7, pp. 1784-1788, 2000. 


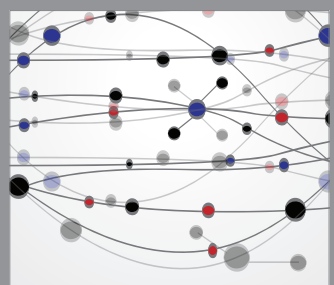

The Scientific World Journal
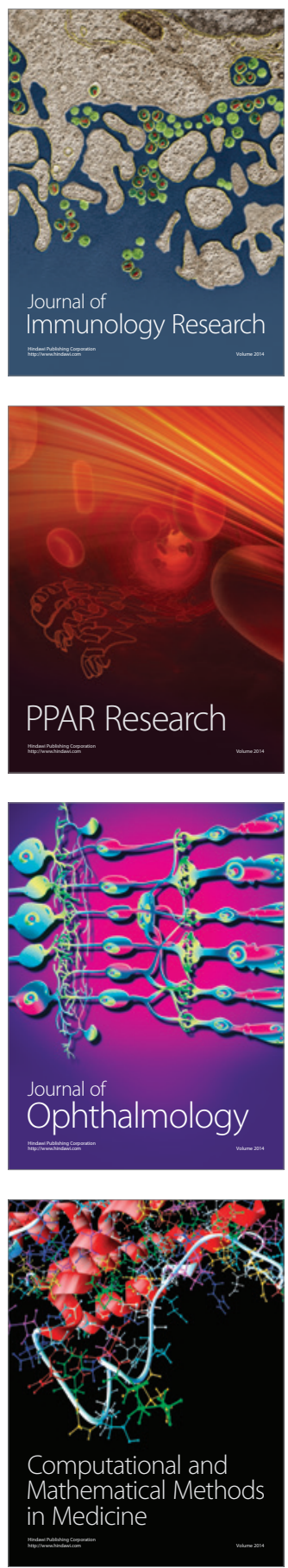

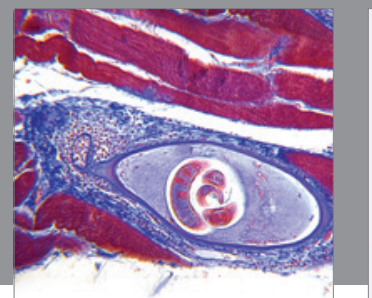

Gastroenterology

Research and Practice
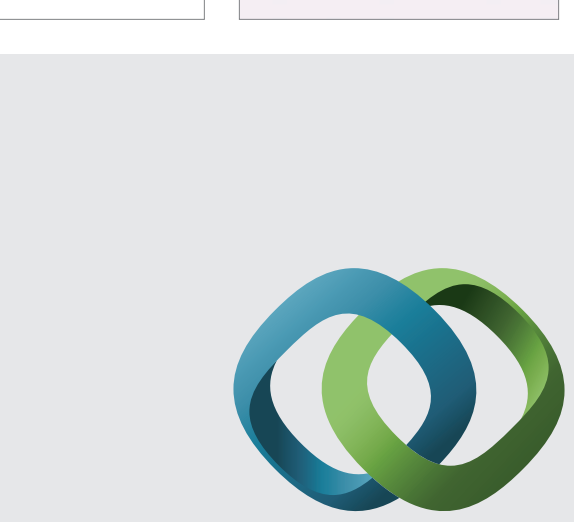

\section{Hindawi}

Submit your manuscripts at

http://www.hindawi.com
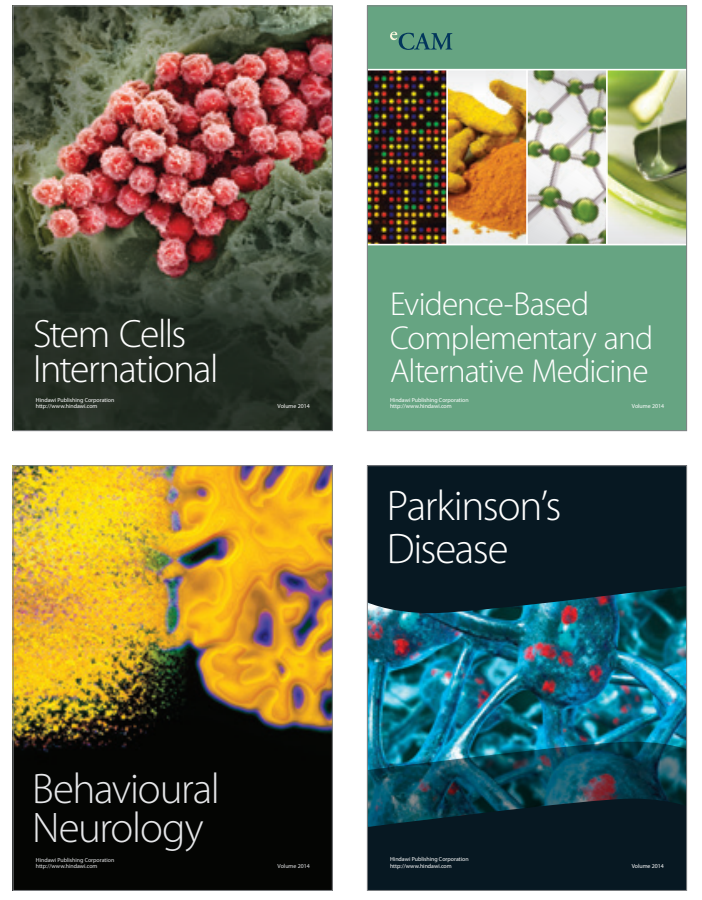
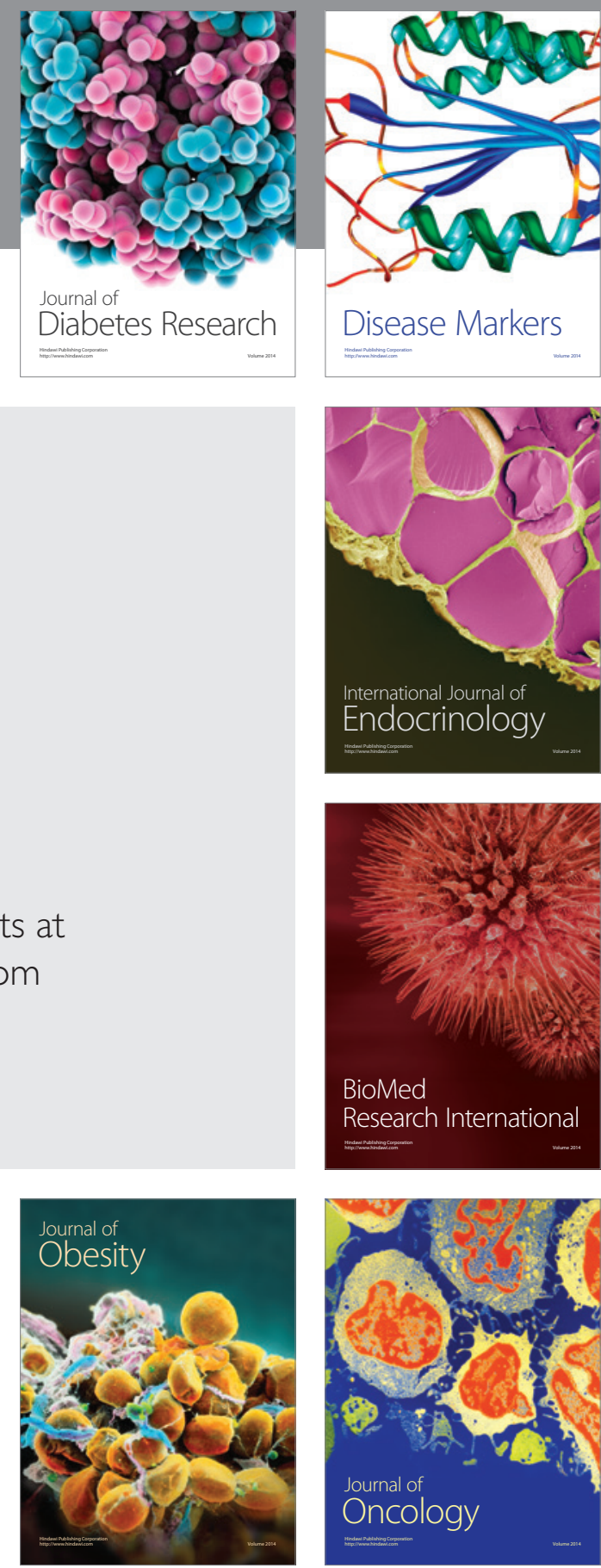

Disease Markers
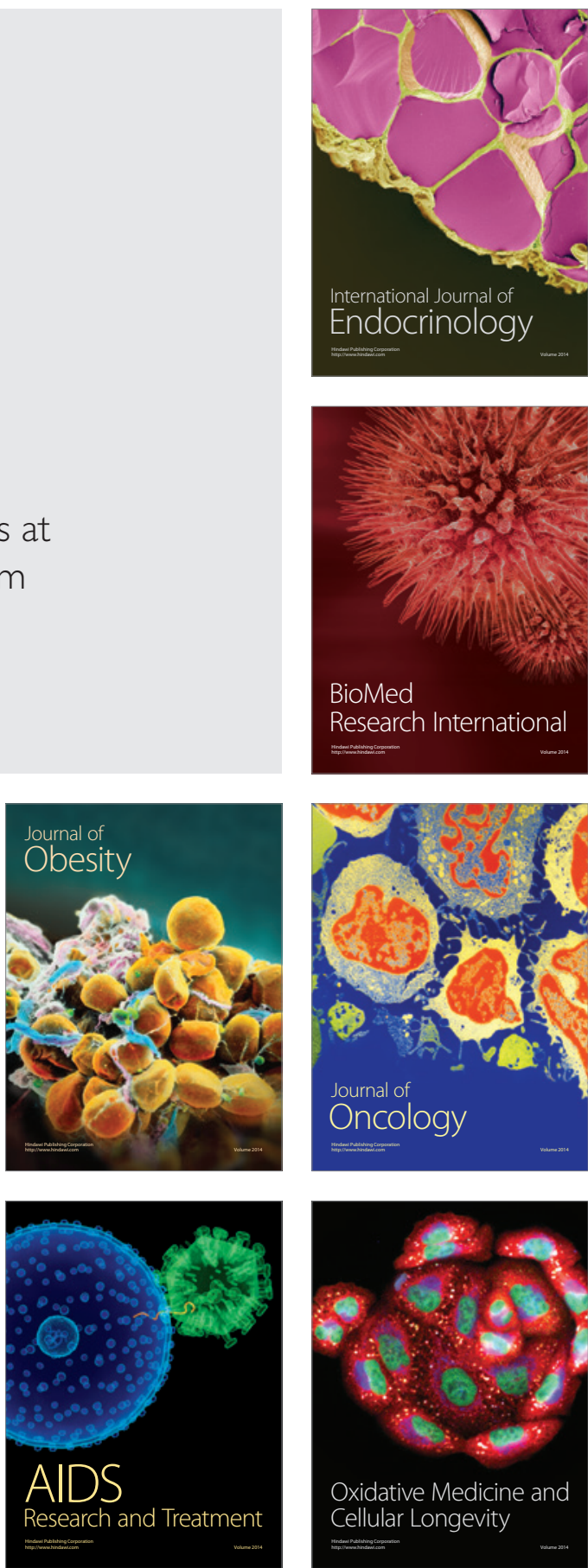